\title{
Intensive care medicine in 2050: the future of ICU treatments
}

\author{
Jean-Louis Vincent ${ }^{1 *} \mathbb{D}$, Arthur S. Slutsky ${ }^{2,3}$ and Luciano Gattinoni ${ }^{4}$
}

(C) 2016 Springer-Verlag Berlin Heidelberg and ESICM

It's unlikely that any of the three of us will still be around, but we can imagine how our children would describe their experience if they were admitted to the ICU in 2050. Here is what they might say:

"The hospital is definitely smaller than in the past with lots of ICU beds, but very few other beds. And, it looks more like a five-star hotel than a hospital, with nice shops and restaurants in the lobby-it makes you forget you're in hospital. My ICU room is fantastic! Really spacious, with a comfortable sofa for my family who can visit anytime. On the wall, there's an enormous screen, which replaces all the small, noisy monitors I remember seeing in photos my dad had of his unit way back 40 years ago. A lot of the time the screen is blank, but it displays alarms when anything goes wrong and can show anything you want to see at the push of a button: trends in variables, all kinds of curves, laboratory results, X-rays, consultant's notes, you name it. There's also a really cool system that teaches me all about my medical condition and that can connect me with other patients who have similar medical issues. The computers that control this can somehow link me to patients around the world who have similar values, expectations, and concerns to mine. And, finally, if you press the red button, a nurse immediately appears on the screen over any other data display, asking what you need or want!

In the past, I remember the phlebotomist used to visit regularly to take blood for various tests, but now I've got a percutaneous sensor that measures almost every lab test. Everything seems to be non-invasive. I remember all the tubes and lines that patients used to have to monitor their heart rate and function-I just have straps around

\footnotetext{
*Correspondence: jlvincent@intensive.org

1 Department of Intensive Care, Erasme University Hospital, Unversité Libre de Bruxelles, Route de Lennik 808, 1070 Brussels, Belgium Full author information is available at the end of the article
}

my thorax and probes on my feet, which the doctors use to continuously monitor my cardiovascular system, and a video-laser system to assess changes in my microcirculation. There's even a metabolic probe to assess peripheral cell oxygenation and viability of my major organs. When I was in shock, my son told me the bed kept moving to assess how much fluid I needed while my cardiac response was monitored by the probes. There was even a closed-loop system driven by a computer that continued to deliver fluid challenges as long as my cardiac output was responsive, combining it with titration of vasopressors and inotropic agents.

I remember lots of my dad's patients used to have tubes in their trachea to help them breath, but they hardly use those anymore! I need help breathing, but just have a special helmet, which is very comfortable. In really severe, acute cases, there's an intubating robot that can insert an endotracheal tube already connected to the respirator, which sits out of the way in the closet! I'm also receiving extracorporeal lung/renal/metabolic support-this is an impressive system that, by passing my blood through an external system, oxygenates it and cleans it of $\mathrm{CO}_{2}$, urea, potassium, inflammatory mediators, and other products that my failing kidneys can no longer eliminate, and replaces them with a caloric mixture including specialized nutrients, all in one smooth passage.

A weird-looking machine just scanned my chest for about a minute and then showed these amazing pictures and videos of my lungs on that huge screen. Not only that, but the pictures were in 3D, so I could see my lungs from inside and out, and how they changed as I breathed! I understand that it's way better than the huge, old, clunky CT and MRI machines of the past, and it provides the interpretation immediately, without what I think used to be called radiologists. They then gave me this pill to treat my lung condition and, using a special wand, they were able to make the medication deliver the

\section{伨


medicine specifically to the diseased parts of my lung, and thus were able to decrease the side effects to the rest of my body!

They do quite a lot of surgical procedures here, but there are hardly any surgeons: all surgery is done remotely with surgeons only present in case of exceptional technical breakdown. That means that one surgeon can operate in several different institutions, and from anywhere. Actually, there are few doctors here altogether, and you never know where they really are when they appear on the screen. Dermatologists look at the pictures that are sent to them, and bronchoscopies, like endovascular procedures, are done by robots. Infectious disease specialists may supervise protocols, but computer systems guide the antibiotic choices and appropriate doses on a daily basis. One of the first treatments I was given on admission was a series of vaccinations against resistant organisms. There are also fewer nurses than in the past, but they are very well trained; they follow protocols and regularly practice and rehearse on simulators.

You might think this all sounds a bit inhuman, but it's not at all. Although there are fewer doctors doing procedures and interventions, the ones that are present are much more available to talk to you, to explain, answer questions, and reassure me and my family. After all, they tell us that the word "doctor" comes from the Latin word docere that means "to teach" and that is now their primary role. In fact, one of the doctors explained to me how my treatment was specifically tailored precisely for me. Somehow they match the therapies I receive to my genetic code so that I get the optimal therapy with the minimal side effects. And apparently, they have this huge database of all the patients' results and outcomes for the past 25 years, so that they can see if anyone else had the weird syndrome I had, and then figure out the best way to treat it. I was also happy to sign a form that all patients sign, agreeing that virtually any treatment I get will be part of a clinical trial, and that all major hospitals are linked as part of a clinical trials network. Apparently, this approach has really advanced medical care. Clinical trials that used to take years are now done in the matter of a few weeks, and the results can be better individualized. They call this large network-based medicine and it replaced the old evidence-based medicine.

Of course, there are still some disciplines where human contact is very important, like obstetrics and pediatrics... and intensive care. But it's certainly well organized-I've been really impressed-physician assistants are available to complement the other staff... and there are even robots that can tell stories or otherwise distract and encourage the patients!

The older doctors and nurses tell us that in the past ICU patients were often sedated, and some even paralyzed. That's really difficult to believe. Today, only alcoholic patients may need sedatives (unless they give them some alcohol, as they say it makes more sense). And bed rest is no longer considered a benefit-patients are only allowed to stay in bed at night, unless you're in shock or in coma. As soon as my shock was resolved, a physiotherapist introduced me to Jim (or is it Gym?), my personalized robot, and explained how "he" would help me exercise and walk with me-we even get out into the landscaped garden surrounding the ICU when the weather is good!

Anyway, I've got to go-they're calling me for my lung transplant, using a lung made from my own stem cells! Speak later."

\section{Author details \\ ${ }^{1}$ Department of Intensive Care, Erasme University Hospital, Unversité Libre de Bruxelles, Route de Lennik 808, 1070 Brussels, Belgium. ${ }^{2}$ Keenan Research Center for Biomedical Science, Li Ka Shing Knowledge Institute, St. Michael's Hospital, Toronto, ON M5B 1W8, Canada. ${ }^{3}$ Interdepartmental Division of Criti- cal Care Medicine, University of Toronto, Toronto, Canada. ${ }^{4}$ Department of Anesthesiology, Emergency and Intensive Care Medicine, University of Göt- tingen, 37075 Göttingen, Germany.}

Compliance with ethical standards

Conflicts of interest

The authors have no conflicts of interest to declare related to this article.

Published online: 4 October 2016 